\title{
The contribution of B cells to transplantation tolerance
}

\author{
Luis Graca
}

Instituto de Medicina Molecular, Faculdade de Medicina, Universidade de Lisboa, Lisbon, Portugal.

\begin{abstract}
Since it was shown in the early 1950 s that it is possible to induce transplantation tolerance in neonates, immune tolerance strategies have been actively pursued. It was found that $T$ cells play a critical role in graft rejection, but can also be major players in mediating transplantation tolerance. Consequently, many experimental systems focused on T cells, often with a complete exclusion of B cells from in vivo animal models. It is now becoming clear that in addition to $T$ cells, B cells can mediate graft rejection and transplantation tolerance. In this issue of the $J \mathrm{Cl}$, Khiew et al. investigated the contribution of alloreactive B cells to transplantation tolerance using a mouse cardiac transplantation model. The authors revealed a distinct tolerant $B$ cell phenotype possessing the ability to suppress naive B cells. These data lead to a better understanding of B cell contributions to transplantation tolerance, and may inform the development of future immune tolerance protocols.
\end{abstract}

\section{Tolerance-inducing protocols}

Therapeutic induction of immune tolerance has been heralded has the holy grail of transplantation, since the pioneering experiments of Billingham, Brent, and Medawar showing it is possible to achieve "actively acquired tolerance" to allografts (1). Since those days, several toleranceinducing protocols have been developed based on different approaches. For instance, it was shown that it is possible to achieve transplantation tolerance through induction of hematopoietic chimerism (i.e., the coexistence of hematopoietic cells of donor and host origin) following hematopoietic stem cell transplantation concomitant with solid organ transplantation (2). In addition, long-term graft acceptance can also be obtained in the absence of immunosuppression following a brief course of treatment with monoclonal antibodies (mAbs) targeting coreceptor (i.e., CD4) or costimulatory (i.e., CD154 - also known as CD40-ligand) molecules (3). Those protocols can lead to transplantation tolerance without compromising immunocompetence; the immune system can readily mount an immune response against other antigens, namely rejecting a transplant from a different donor, while remaining tolerant to subsequent exposure of the tolerated antigens (for instance, a new transplant genetically identical to the tolerated graft) (Figure 1A).

When the cellular and molecular mechanisms underlying transplantation tolerance were initially dissected there was an understandable focus on the role of $\mathrm{T}$ cells, as those cells are the main players in graft rejection. It soon became apparent, however, that the different toleranceinducing protocols were based on distinct cellular mechanisms. Mixed hematopoietic chimerism was shown to rely primarily on alloreactive $\mathrm{T}$ cell elimination (4, 5). The resulting immune system, purged of alloreactive $\mathrm{T}$ cell clones, could not mount a response against the allograft, but remained competent to respond to unrelated antigens. In contrast, tolerance induced with mAbs targeting coreceptor and costimulatory molecules was shown to

Related Article: p. 3453

Conflict of interest: The author has declared that no conflict of interest exists.

Copyright: () 2020, American Society for Clinical Investigation.

Reference information: J Clin Invest. 2020;130(7):3406-3408. https://doi.org/10.1172/JCl138122.

induce an incomplete deletion of alloreactive clones (although critical for tolerance induction), together with the emergence of Tregs that could actively maintain the tolerant state (6). The experimental systems adopted to dissect the molecular mechanisms involved under those different immune tolerance protocols were often based on a reductionist approach to allow detailed investigation of alloreactive $\mathrm{T}$ cells. As a consequence, many approaches (including some approaches pursued by our group) relied on in vivo models devoid of $B$ cells (namely adoptive $T$ cell transfers into immunodeficient hosts), complementing experiments performed in WT animals. Therefore, the understanding of B cell contributions to transplantation tolerance advanced at a slower pace. However, in addition to the $\mathrm{T}$ cell contribution to graft rejection, it became increasingly recognized that donor-specific antibodies (DSAs) constitute a major hurdle precluding long-term graft survival in the clinic (7). Therefore, a more careful consideration of the participation of $\mathrm{B}$ cells in long-term transplant rejection and tolerance is needed.

\section{Participation of B cells in long- term transplantation}

Khiew and colleagues investigated the donor-specific B cell compartment in transplantation tolerance induced through costimulation blockade with anti-CD154 mAbs and donor cell transfusion at the time of heart transplantation (8). DSAs targeting MHC molecules are among the most clinically important. The authors, therefore, used tetramers to follow the fate of MHC-specific B cells as transplantation tolerance became established and was maintained. The impact of this tolerance-inducing protocol, based on costimulation blockade, on alloreactive $\mathrm{T}$ cells has been thoroughly investigated; it was shown that tolerance requires elimination of many donor-specific $\mathrm{T}$ cells through activation-induced cell death $(9,10)$, with some antigen-specific $\mathrm{CD} 4^{+} \mathrm{T}$ cells that survive deletion and acquire regulatory proper- 
A

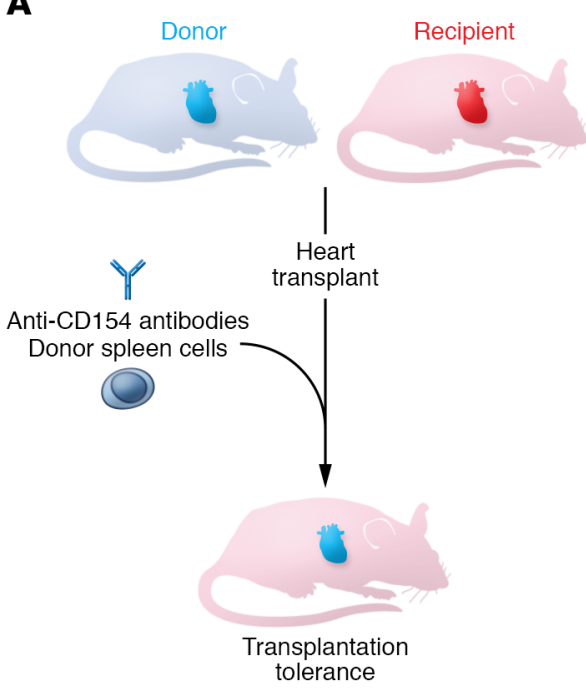

B

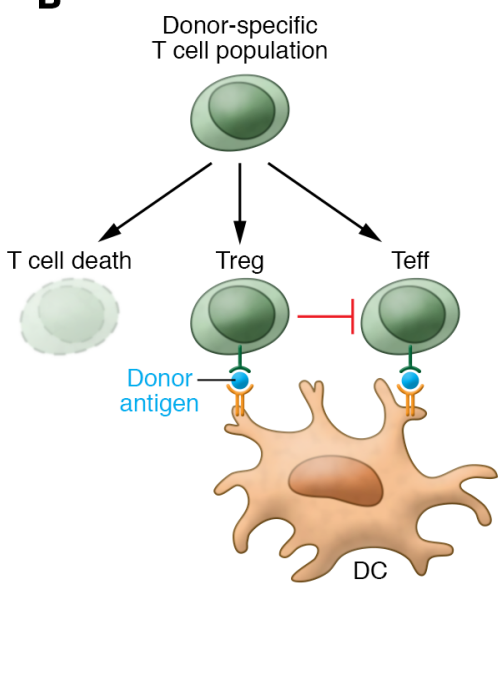

C

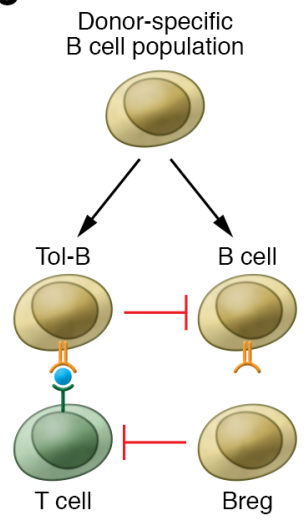

Figure 1. The impact of transplantation tolerance on $\mathbf{T}$ and B cell populations. (A) In murine heart transplantation, treatment with anti-CD154 antibody and donor spleen cells (costimulation blockade) at the time of transplantation leads to donor-specific tolerance. The antigen-specific tolerance is a consequence of changes in T and B cell populations. (B) Many donor-specific T cells are deleted. Other T cells acquire regulatory function (Treg) endowing them with the ability to suppress remaining donor-specific T cells (Teff). (C) B cells are mainly converted into a tolerant phenotype (Tol-B), distinct from what has been described for regulatory B (Breg) cells. Unlike Breg cells, Tol-B cells do not directly suppress T cells. However, Tol-B cells can effectively suppress other B cells sharing the same donor-antigen specificity. Overall, changes in donor-specific $T$ and B cell populations allow suppression of immune responses targeting the donor antigens, without preventing protective immune responses against unrelated antigens. DC, dendritic cell.

ties being able to maintain the tolerant state (Figure $1 \mathrm{~B}$ and ref. 11). In fact, those Treg cells can prevent rejection mediated by adoptively transferred donor-specific $\mathrm{T}$ cells (a demonstration that tolerance is dominant), and can convert some of those $\mathrm{T}$ cells into a new cohort of Treg cells with a suppressive role - a phenomenon termed infectious tolerance (11-13).

One possible mechanism to impose tolerance on the B cell compartment could be $\mathrm{B}$ cell deletion, especially given prior reports using $\mathrm{B}$ cell receptor-knockin (BCR-knockin) mice (with a very large frequency of donor-specific B cells) where deletion has been observed (14). However, the current study has shown that, under physiologic conditions regarding the $\mathrm{B}$ cell clone size, mice that became tolerant to heart transplants with anti-CD154 treatment maintained donor-specific B cell counts (8). Furthermore, donor-specific B cells not only survived, but displayed evidence of antigen-mediated activation, although they failed to differentiate into germinal center (GC) B cells, and failed to contribute to production of DSAs. It was therefore likely that B cells were functionally altered when exposed to antigen stimulation in the absence of a second signal - in this case CD154.

\section{The two-signal model}

It is timely that the study by Khiew et al. (8) is published exactly half a century after the influential two-signal model proposed by Bretscher and Cohn (15). Although the two-signal model was initially proposed in relation to $\mathrm{B}$ cells, it was subsequently extended to T cells (16). In brief, the two-signal model postulates that an immune cell requires antigen recognition (signal 1) and a costimulatory signal (signal 2) to become fully activated toward an effector role. When antigen recognition takes place in the absence of the second signal, the model postulates that the outcome will be deletion or anergy (i.e., functional inactivation).

Khiew et al. (8) observed that antiCD154-induced transplantation tolerance forced B cells to respond to antigen recognition through the BCR in the absence of $\mathrm{CD}^{+} \mathrm{T}$ cell help provided by CD154. As a consequence, $\mathrm{B}$ cells that received signal 1 in the absence of CD154 engagement adopted a functionally impaired, decommissioned phenotype. Those decommissioned B cells could still interact with $\mathrm{CD}^{+} \mathrm{T}$ cells supporting $\mathrm{Tfh}$ differentiation, but failed to fully differentiate into GC B cells and did not produce DSA (Figure $1 \mathrm{C})$. In addition, that decommissioned, unresponsive phenotype was maintained even when the tolerant B (Tol-B) cells were adoptively transferred into a new host, and challenged with allogeneic cells syngeneic with the tolerized transplant. However, the most striking feature of those decommissioned B cells is that they were able to suppress new cohorts of naive B cells, providing those naive $B$ cells were also specific to the same tolerated donor antigens (8). These tolerized B cells display, therefore, characteristics of $\mathrm{B}$ cell-dominant tolerance, by being able to actively suppress naive B cells. It remains unaddressed whether those naive B cells, when exposed to B cell-mediated suppression, can themselves acquire suppressive function, akin to what has been described for $\mathrm{T}$ cells in the process of infectious tolerance.

It is somewhat surprising that the authors did not find evidence for a greater degree of cross-reactivity between epitopes from the tolerated transplant, and epitopes from the third-party graft used to demonstrate the antigen specificity of $\mathrm{B}$ cell tolerance. In fact, it has been observed that dominant transplantation tolerance mediated by $\mathrm{CD} 4^{+}$Treg cells across a full MHC mismatch can also manifest, to a certain degree, toward third-party MHC, presumably due to cross-reactivity (4). 


\section{Distinct B cells}

The detailed mechanism of action of the decommissioned B cells with suppressive properties remains unknown. These B cells, described by Khiew et al., are distinct from other better-characterized suppressive B cells, namely B regulatory (Breg) cells $(17,18)$. Breg cells have a characteristic phenotype, different from the suppressive B cells that emerge following induction of transplantation tolerance. In addition, Breg cells are able to regulate $\mathrm{T}$ cell responses through cytokine production (namely IL-10), while the Tol-B cells studied by Khiew et al. seem to be exclusively able to suppress other B cells and do not produce IL-10. It will also be necessary to investigate the range of conditions leading to induction of these suppressive B cells. The report clearly shows that exposure to donor antigens in the absence of CD154 signaling can lead to suppressive B cells, with tolerance induction with CTLA-4-Ig leading to similar observations. However, it will be important to investigate whether other tolerance-inducing regimens can have identical effects, and whether patients with long-term graft acceptance in the absence of DSA may support the progressive emergence of $B$ cells that restrain humoral responses against the transplanted tissue. While we may still be far from using B cell subsets for clinical monitoring of transplanted patients, it is important to keep in mind that although classically " $\mathrm{T}$ " was the letter for Tolerance and Transplantation, we should keep "B" in the alphabet.

Address correspondence to: Luis Graca, Instituto de Medicina Molecular, Faculdade de Medicina da Universidade de Lisboa, Avenida Egas Moniz, 1649-028 Lisboa, Portugal. Phone: 351.21.796.7624

Email:lgraca@medicina.ulisboa.pt.

1. Billingham RE, Brent L, Medawar PB. Actively acquired tolerance of foreign cells. Nature. 1953;172(4379):603-606

2. Sachs DH, Kawai T, Sykes M. Induction of tolerance through mixed chimerism. Cold Spring Harb Perspect Med. 2014;4(1):a015529.

3. Waldmann H, Adams E, Cobbold S. Reprogramming the immune system: co-receptor blockade as a paradigm for harnessing tolerance mechanisms. Immunol Rev. 2008;223:361-370.

4. Graca L, Le Moine A, Lin CY, Fairchild PJ, Cobbold SP, Waldmann H. Donor-specific transplantation tolerance: the paradoxical behavior of CD $4^{+} \mathrm{CD} 25^{+}$T cells. Proc Natl Acad Sci U S A. 2004;101(27):10122-10126.

5. Kurtz J, Wekerle T, Sykes M. Tolerance in mixed chimerism - a role for regulatory cells? Trends Immunol. 2004;25(10):518-523.

6. Waldmann H, Hilbrands R, Howie D, Cobbold S. Harnessing FOXP3 ${ }^{+}$regulatory T cells for transplantation tolerance. J Clin Invest. 2014;124(4):1439-1445.

7. Loupy A, Lefaucheur C. Antibody-mediated rejection of solid-organ allografts. N Engl JMed. 2018;379(12):1150-1160.
8. Khiew SHW, et al. Transplantation tolerance modifies donor-specific B cell fate to suppress de novo alloreactive B cells. JClin Invest. 2020;130(7):3453-3466.

9. Wells $\mathrm{AD}$, et al. Requirement for T-cell apoptosis in the induction of peripheral transplantation tolerance. Nat Med.1999;5(11):1303-1307.

10. Li Y, Li XC, Zheng XX, Wells AD, Turka LA, Strom TB. Blocking both signal 1 and signal 2 of T-cell activation prevents apoptosis of alloreactive T cells and induction of peripheral allograft tolerance. Nat Med.1999;5(11):1298-1302.

11. Graca L, Honey K, Adams E, Cobbold SP, Waldmann H. Cutting edge: anti-CD154 therapeutic antibodies induce infectious transplantation tolerance. J Immunol. 2000;165(9):4783-4786.

12. Qin S, et al. "Infectious" transplantation tolerance. Science. 1993;259(5097):974-977.

13. Kendal AR, et al. Sustained suppression by Foxp $3^{+}$regulatory $\mathrm{T}$ cells is vital for infectious transplantation tolerance. J Exp Med. 2011;208(10):2043-2053.

14. Li Y, Ma L, Shen J, Chong AS. Peripheral deletion of mature alloreactive B cells induced by costimulation blockade. Proc Natl Acad Sci U S A. 2007;104(29):12093-12098.

15. Bretscher P, Cohn M. A theory of self-nonself discrimination. Science. 1970;169(3950):1042-1049.

16. Cohn M. The wisdom of hindsight. Annu Rev Immunol. 1994;12:1-62.

17. Ding Q, et al. Regulatory B cells are identified by expression of TIM- 1 and can be induced through TIM-1 ligation to promote tolerance in mice. J Clin Invest. 2011;121(9):3645-3656.

18. Shen P, et al. IL-35-producing B cells are critical regulators of immunity during autoimmune and infectious diseases. Nature. 2014;507(7492):366-370. 